\title{
Seizures and Cardiac Tamponade in Long-Standing Severe Hypothyroidism
}

\author{
May Uyking Naranjo and Marjorie Ramos \\ Section of Endocrinology, Diabetes and Metabolism, Department of Medicine \\ St. Luke's Medical Center, Quezon City, Philippines
}

\begin{abstract}
\section{Introduction}

Untreated or unrecognized hypothyroidism may progress to severe decompensation or myxedema coma. This condition is characterized by marked impairment of the central nervous and cardiovascular systems, with a high mortality rate if left untreated. A significant delay in diagnosis may adversely affect the prognosis. Even though sensitive TSH assays are widely available, the recognition and therapy of myxedema coma remains a challenge.
\end{abstract}

We report a patient who presented with a seizure episode and concurrent clinical and echocardiographic evidence of cardiac tamponade caused by severe hypothyroidism. The symptoms improved following immediate tube pericardiostomy and thyroxine hormone replacement. The classic symptoms of severe hypothyroidism are now rarely seen given the wide availability of thyrotropin (TSH) assays and thyroid hormone tablets. The simultaneous occurrence of both symptoms during a single admission has not been previously reported in literature.

Keywords: severe hypothyroidism, myxedema coma, seizure, pericardial tamponade

High-dose thyroid hormone replacement is typically recommended for the treatment of myxedema coma. However, the most effective choice whether to use T3, T4 or a combination and amount of thyroid hormone therapy is still controversial. ${ }^{1}$ In this article, we report a patient who presented with a rare manifestation of untreated severe hypothyroidism, who subsequently underwent low-dose levothyroxine therapy and pericardiostomy with satisfactory clinical outcome.

\section{Case}

A 69-year old Filipino woman presented to us with seizures and pericardial tamponade. Seven months prior to admission, she was noted to have progressive fatigue and lethargy, with inability to perform activities of daily living. She had exertional dyspnea, waxing and waning bipedal edema, decreased bowel movement frequency ( 2 to 3 times per month) and cold intolerance. She had no apparent paroxysmal nocturnal dyspnea, orthopnea, oliguria, chest pain, syncope or palpitations. On the day of

ISSN 0857-1074

Copyright $(2011$ by the JAFES

Received September 15, 2011. Accepted October 18, 2011. admission, as she was seated waiting for her out-patient clinic consult, she was found to have sudden upward rolling of the eyeballs followed by stiffening of the extremities. There was associated urinary incontinence and loss of consciousness for approximately 5 to 10 seconds. There was no recollection of the event afterward. She was subsequently admitted. On review of her medical history, she had total thyroidectomy in 2007 (Four years PTC) for Hashimoto's thyroiditis, but was poorly compliant with thyroid hormone replacement and stopped taking the medication since October 2010. She had no known history of ischemic heart disease, diabetes mellitus or hypertension.

On examination, the patient was wheelchair borne, conscious, and able to follow commands. She had occasional disorientation, slow and disorganized thought process She had a hoarse voice, and her facial features appeared puffy (Figure 1A). She had dry skin, coarse hair and dystrophic nails (Figure $1 \mathrm{~B}$ and $1 \mathrm{C}$ ). She had a visible thyroidectomy scar. Her vital signs revealed a temperature of $35^{\circ} \mathrm{C} /$ tympanic, a cardiac rate of 55 beats/minute, and a blood pressure of $120 / 80 \mathrm{~mm} \mathrm{Hg}$. She had muffled heart sounds and bibasal rales on auscultation. Her abdomen was soft and flabby, with hypoactive bowel sounds and mild tenderness on all quadrants. She had grade 2 bipedal non-pitting edema. Neurological findings showed a motor strength of $3 / 5$ on all extremities and generalized hyporeflexia, with no sensory deficits and negative Babinski's sign

At the emergency room, a primary neurologic cause was initially considered. Cranial CT scan showed cerebro-

Corresponding author: May Uyking Naranjo,MD

Diabetes, Thyroid and Endocrine Center

St. Luke's Medical Center

CHBC 12th Floor, North Tower

279 E. Rodriguez, Quezon City, Philippines

Tel.No. (632)-7230101 local 5210

Mobile No.: 09177005788

Email: may22uyking@yahoo.com 
cerebellar volume loss but no evidence of acute infarction or intracranial hemorrhage. Electroencephalogram (EEG) showed mild diffuse slowing of activities. A standing order of diazepam $10 \mathrm{mg}$ IV was given for frank seizure.

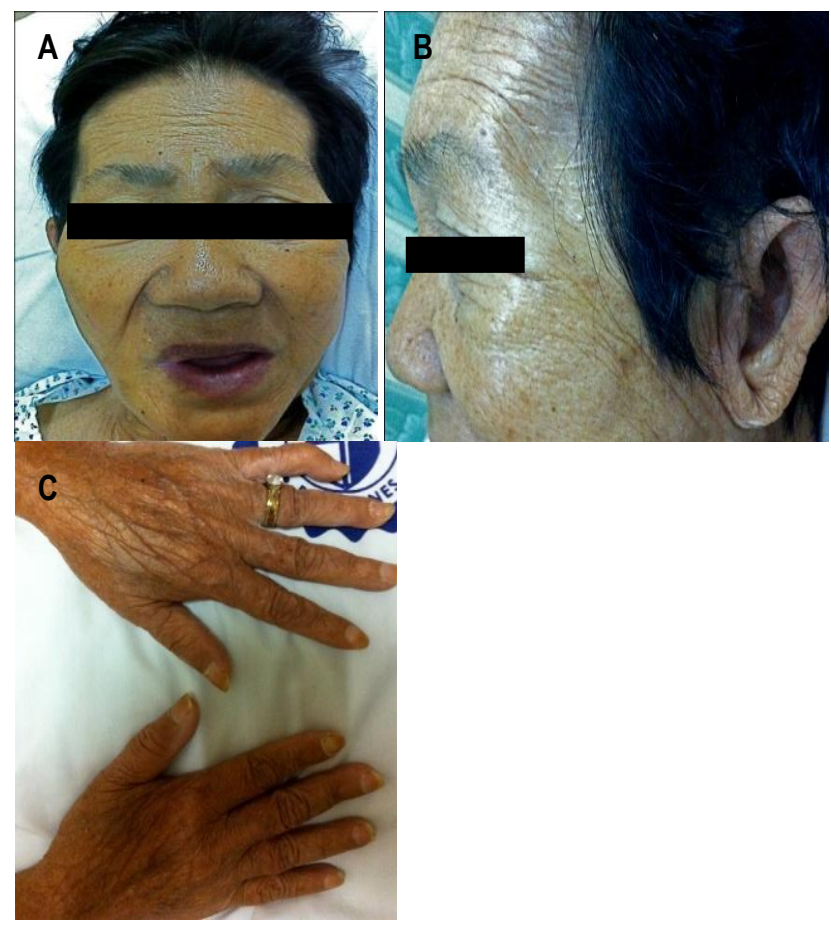

Figure 1. On examination, the patient had coarsened features, sallow complexion and puffy appearance (A). She had non-pitting, waxy, dry edema in the subcutaneous tissues around the eyes and ears, due to the deposition of mucopolysaccharides within the dermis (B). Hypercarotenemia gives the skin a yellow tint $(C)$. The dystrophic fingernails are also notable.

Table 1 lists the relevant laboratory results. Thyroid function testing subsequently confirmed severe hypothyroidism, with elevated TSH $(163.10 \mathrm{mU} / \mathrm{L})$ and barely detectable total triiodothyronine (T3) $(<0.006$ $\mathrm{nmol} / \mathrm{L})$ and total thyroxine (T4) $(<12.87 \mathrm{nmol} / \mathrm{L})$ levels. Serum electrolytes revealed a low normal serum sodium level $(137 \mathrm{mmol} / \mathrm{L})$. She had leukopenia with no apparent focus of infection. She also had hypercholesterolemia (LDL-C $6.93 \mathrm{mmol} / \mathrm{L}$ ) and elevated total creatinine kinase (CK-total $727 \mathrm{U} / \mathrm{L}$ ). An EMG-NCV revealed focal median neuropathy at the level of both wrists compatible with carpal tunnel syndrome. Plain film of the abdomen showed ileus with fecal retention. Chest X-ray revealed cardiomegaly with no evidence of pulmonary congestion (Figure 2). Electrocardiogram on admission showed sinus arrhythmia with a heart rate of 86 beats/min and diffuse flattened $\mathrm{T}$ waves in all leads.

Levothyroxine was initially started at $25 \mathrm{mcg}$ daily, and was then increased to $37.5 \mathrm{mcg}$ daily after the third day. Because of the possibility of concomitant hypocortisolism, the patient was given hydrocortisone $100 \mathrm{mg}$ intravenously every eight hours after a serum cortisol level was obtained.
Table 1. Pertinent Laboratory Examinations

\begin{tabular}{lcccc}
\hline \multicolumn{1}{c}{ Variable } & $\begin{array}{c}\text { On } \\
\text { Admission }\end{array}$ & $\begin{array}{c}\mathbf{4}^{\text {th }} \\
\text { week }\end{array}$ & $\begin{array}{c}\mathbf{1 2}^{\text {th }} \\
\text { week }\end{array}$ & $\begin{array}{c}\text { Normal } \\
\text { Range(SI Unit) }\end{array}$ \\
\hline Hemoglobin $(\mathrm{g} / \mathrm{L})$ & 110 & 106 & & $116-155$ \\
White blood cell $\left(10^{9} / \mathrm{L}\right)$ & 3440 & 3650 & & $4800-10800$ \\
Sodium $(\mathrm{mmol} / \mathrm{L})$ & 137 & 142 & & $136-145$ \\
Potassium $(\mathrm{mmol} / \mathrm{L})$ & 3.6 & & $3.5-5.1$ \\
Creatinine $(\mu \mathrm{mol} / \mathrm{L})$ & 114.9 & & & $0.53-106$ \\
Cortisol(baseline) $(\mathrm{nmol} / \mathrm{L})$ & 278.6 & & & \\
Cortisol (30mins) & 535.2 & & & \\
Cortisol (60mins) & 435.9 & & & $1.10-10.10$. \\
ACTH (pmol/L) & 1.89 & & 0.45 & $0.35-5.50$ \\
TSH mU/L & 163.10 & & & $0.01-0.027$ \\
Total Triidothyronine (nmol/L) & $<0.0061$ & 74.24 & & $57.9-160.87$ \\
Total Thyroxine (nmol/L) & $<12.87$ & & 12.61 & $11.45-22.65$ \\
Free Thyroxine(pmol/L) & & & $4.10-5.88$ \\
Glucose (mmol/L) & 4.4 & 8.62 & 3.96 & $<5.18$ \\
Cholesterol (mmol/L) & 9.04 & & 1.34 & $0.22-2.26$ \\
Triglycerides (mmol/L) & 1.68 & & 0.59 & $>0.90$ \\
HDL cholesterol (mmol/L) & 0.67 & & 2.82 & $0-4.11$ \\
LDL cholesterol (mmol/L) & 6.93 & & & $26-192$ \\
Total Creatinine Kinase (U/L) & 727 & & & \\
\hline & & &
\end{tabular}

A 2D-echocardiogram showed left ventricular hypertrophy, normal right ventricular dimension with evidence of diastolic collapse noted at the right ventricular outflow tract (RVOT), normal right and left atrium, moderate pericardial effusion mostly noted in the lateral portion of the left ventricular free wall with signs of tamponade physiology (Figure 3). A tube pericardiostomy drained $890 \mathrm{~mL}$ of straw-colored pericardial fluid. The pericardiostomy tube was removed on the $3^{\text {rd }}$ postoperative day. Cytological examination of the pericardial fluid did not reveal bacterial or viral infection. There were no malignant cells or granulomas seen on examination of the pericardial tissue.

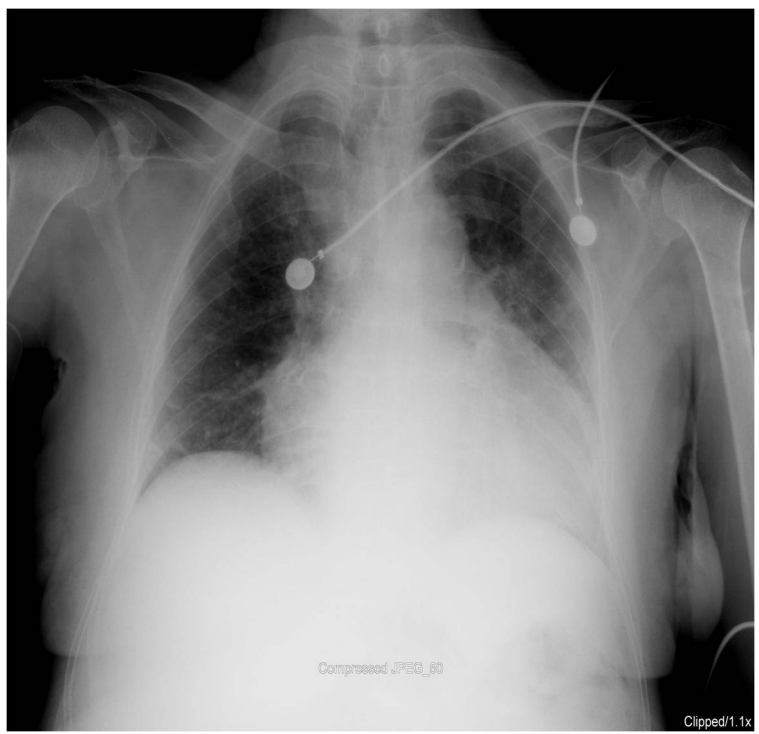

Figure 2. Chest radiograph of the patient showing cardiomegaly.

The patient's subsequent hospital course was uneventful. She remained seizure-free throughout; her breathing improved and her bipedal edema decreased. Hydrocortisone was tapered and discontinued upon note of adequate adrenal reserve from an ACTH stimulation test. The dose of levothyroxine was gradually increased to $100 \mathrm{mcg} /$ day by the fourth week. 


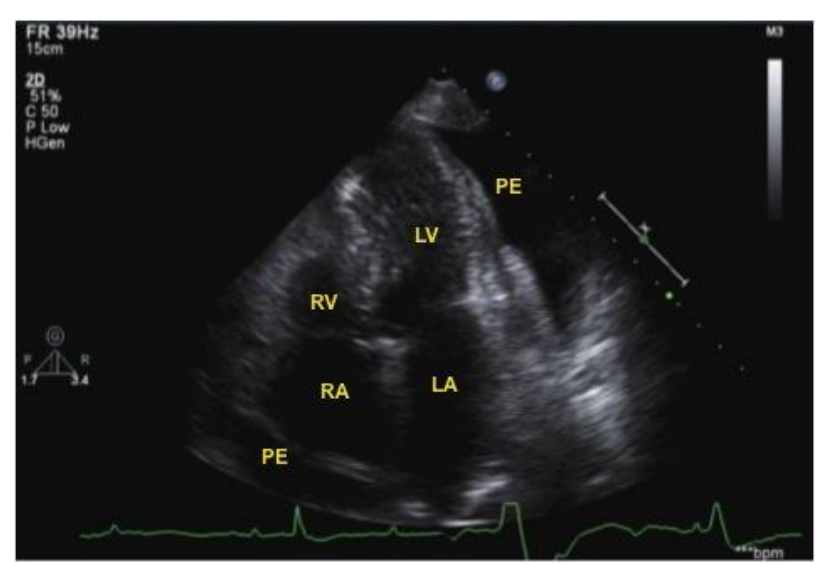

Figure 3. Apical four-chamber echocardiogram showing echo-free space consistent with moderate pericardial effusion: $P E$, pericardial effusion; $R A$, right atrium; $L A$, left atrium; $R V$, right ventricle; $L V$, left ventricle.

Four weeks after hospital discharge, the patient was able to ambulate with minimal assistance (Figure 4). Her bowel movement frequency improved to 2 to 3 times per week. TSH level decreased to $74.24 \mathrm{mU} / \mathrm{L}$, while free T4 was 8.62 pmol/L. Repeat echocardiogram showed no evidence of diastolic collapse and resolution of pericardial effusion. Lipid profile and TSH level normalized after twelve weeks (Table 1).

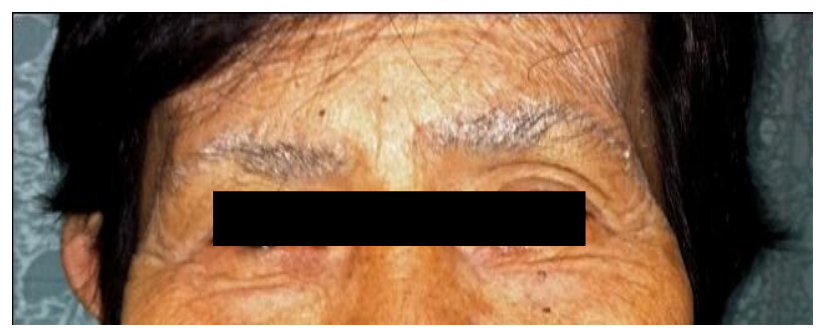

Figure 4. Four weeks after discharge, there was a significant improvement in her facial puffiness. Note the loss of the lateral third of the eyebrows, called Queen Anne's sign.

\section{Discussion}

Myxedema coma, a term used interchangeably with severe hypothyroidism, is an endocrine emergency. This is a potentially lethal condition that requires aggressive treatment. The mortality rates may be as high as 25 to $60 \%$ even with the best possible treatment. ${ }^{2-3}$ The true incidence is unknown, with only over 300 cases reported in literature. ${ }^{4-5}$ Environmental factors (such as low ambient temperature), infection, cerebrovascular accidents, drugs (particularly tranquilizers, anesthetics, sedatives, narcotics and lithium), and consumption of raw bok choy, may precipitate myxedema crisis. ${ }^{6}$ In our patient, the condition resulted from long-standing untreated hypothyroidism.

Essentially, thyroid hormones affect every organ system, including the central and peripheral nervous systems. The absence of coma does not rule out the condition. Some patients manifest with lesser degrees of altered consciousness, like confusion with lethargy. Other neurologic complications include cranial nerve palsies, hoarseness, myopathy, neuropathy, carpal tunnel syndrome, reflex changes, ataxia, and psychotic features, the so-called "myxedema madness."7 About 25\% of patients have focal or generalized seizures. ${ }^{2}$ The exact mechanisms are unclear. The possible mechanism of seizure in our patient may be cerebral hypoxia. Brain function is affected by reduction in cerebral blood flow and oxygen delivery, deficiency of T4 and T3, and glucose consumption; as such, it is likely all these factors are probably involved. EEG findings are nonspecific, with slowing and decreased amplitude and rarely with triphasic waves. ${ }^{8}$

Pericardial effusion in hypothyroidism is related to the severity and duration of the disease. Its incidence is reported to be at $3 \%$ in early mild stage to $80 \%$ when myxedema is present. ${ }^{9-10}$ The occurrence of cardiac tamponade in hypothyroidism is uncommon due to the slow accumulation of fluid and the remarkable distensibility of the pericardium. ${ }^{11}$ The classic signs of hypotension, muffled heart sounds, jugular vein distention and pulsus paradoxus are not always present. ${ }^{12}$ The compensatory tachycardia in a patient with tamponade is usually absent in hypothyroidism, as in our case. Many hypothyroid patients have high serum CK concentrations. The isoenzyme distribution is almost completely $\mathrm{MM}$, with less than $4 \%$ constituting $\mathrm{MB}$, indicative of skeletal muscle origin. ${ }^{13}$ ECG may reveal lowamplitude QRS complexes, prolonged QT interval, and flattened or inverted $\mathrm{T}$ waves. Echocardiography remains the most reliable diagnostic modality with exceptionally high specificity and sensitivity. ${ }^{14}$ Echocardiographic features of tamponade include chamber collapse and reciprocal changes in left and right ventricular volumes with respiration. ${ }^{12}$ Pericardial fluid exceeding $25-50 \mathrm{ml}$ is seen as an echo-free space through-out the cardiac cycle signifying pericardial effusion.

Thyroid hormone therapy is the cornerstone of treatment of patients with myxedema coma. High dose thyroid hormone replacement has been typically recommended. However, because of the paucity of cases, there are no clinical trials comparing the efficacy of different treatment regimens, and the optimal therapy has yet to be determined. Some experts favor administration of T3 because of its greater biologic activity and more rapid onset of action; others prefer a combination of T4 and T3. Whether patients with myxedema coma should be treated with T4, T3, or both, is still controversial. ${ }^{1}$

Selected cases of myxedema coma can be safely treated with thyroid hormone at doses lower than conventionally recommended. ${ }^{15}$ In a hypothyroid patient with cardiac tamponade, the recommended therapeutic regimen is levothyroxine at an initial low dose $(25 \mathrm{mcg} /$ day), to be 
increased gradually. Higher doses may precipitate a cardiac event that may lead to further decompensation towards tamponade. ${ }^{16}$ In our case, the treatment given was low dose levothyroxine at $25 \mathrm{mcg} /$ day, which was increased gradually to $100 \mathrm{mcg} /$ day by the fourth week. The patient recovered without sequelae.

Our patient presented with the classic manifestations and laboratory abnormalities of severe hypothyroidism. However, the seizure episode with concomitant cardiac tamponade represents an extreme form of complicated hypothyroidism. Early recognition and prompt therapy are essential. In light of the possible adrenal insufficiency due to polyglandular autoimmune syndromes or hypothalamic-pituitary compromise, many experts recommend empiric treatment with stress-dose glucocorticoids (hydrocortisone $100 \mathrm{mg}$ IV every 6-8 hours) until definitive ACTH stimulatory testing is performed and concomitant adrenal insufficiency is ruled out. Pericardial effusion may be easily reversed with thyroid hormone replacement, but tube pericardiostomy is necessary when tamponade or impending tamponade develops.

Lastly, supportive measures, including treatment in an intensive care unit setting; ventilatory support, if indicated; judicious administration of intravenous fluids, electrolytes and glucose; correction of hypothermia by passive rewarming with ordinary blankets and warm ambient temperature; avoidance of hypnotics and sedatives; and treatment of any underlying infection; are also important in the management of the severely hypothyroid patient.

\section{References}

1. Kwaku MP, Burman KD. Myxedema coma. J Intensive Care Med. 2007;22(4):224-31.

2. Wartofsky L. Myxoedema coma. Endocrinol Metab Clin N Am. 2006:35:687-98

3. Rodríguez I, Fluiters E, Pérez-Méndez LF et al. Factors associated with mortality of patients with myxoedema coma: Prospective study in 11 cases treated in a single institution. J Endocrinol. 2004;180(2):347-50.

4. Wall CR. Myxedema coma: Diagnosis and treatment. Am Fam Phys. 2000; 62:2485-90

5. Nicoloff JT, LoPresti JS. Myxedema coma: A form of decompensated hypothyroidism. Endocrinol Metab Clin North Am. 1993;22:279-90.

6. Chu M, Seltzer TM. Myxedema coma induced by ingestion of raw bok choy. N Engl J Med. 2010;362:1945-6.

7. Westphal SA. Unusual presentations of hypothyroidism. Am J Intensive Care Med. 2007;22:224.

8. Haupt M, Kurz A. Reversibility of dementia in hypothyroidism. J Neurol. 1993; 240:333.

9. Hardisty CA, Naik DR, Munro DS. Pericardial effusion in hypothyroidism. Clin Endocrinol. 1980;13:349-54.

10. Kabadi UM, Kumar SP. Pericardial effusion in primary hypothyroidism. Am Heart J. 1990;120:1393-5.

11. Braunwald E, Lorell BH. Pericardial Disease. In: Heart Disease: A Textbook of Cardiovascular Medicine, edited by Eugene Braunwald, 1465-516. Philadelphia: WB Saunders, 1992.

12. Hoit BD. Cardiac Tamponade. Uptodate, edited by Gersch, BJ, Hoekstra J, Downey BC. September 2009

13. Klein I, Mantell P, Parker M, Levey GS. Resolution of abnormal muscle enzymes in hypothyroidism. Am J Med Sci. 1980;279:159-62.

14. Horwitz MS, Schultz CS, Stinson EB et al. Sensitivity and specificity of echocardiographic diagnosis of pericardial effusion. Circulation. $1974 ; 50: 239-47$

15. Fujiyoshi A, Yamamoto T. Two cases of myxedema coma successfully treated with low-dose thyroid hormone replacement. Journal of Japanese Association for Acute Medicine. 2000;11:22-6.

16. Rechad M, Cassim, Trentini AP et al. Pericardial effusion with cardiac tamponade as a form of presentation of primary hypothyroidism. Archives of Brazil Cardiology. 2002;78:583-5.

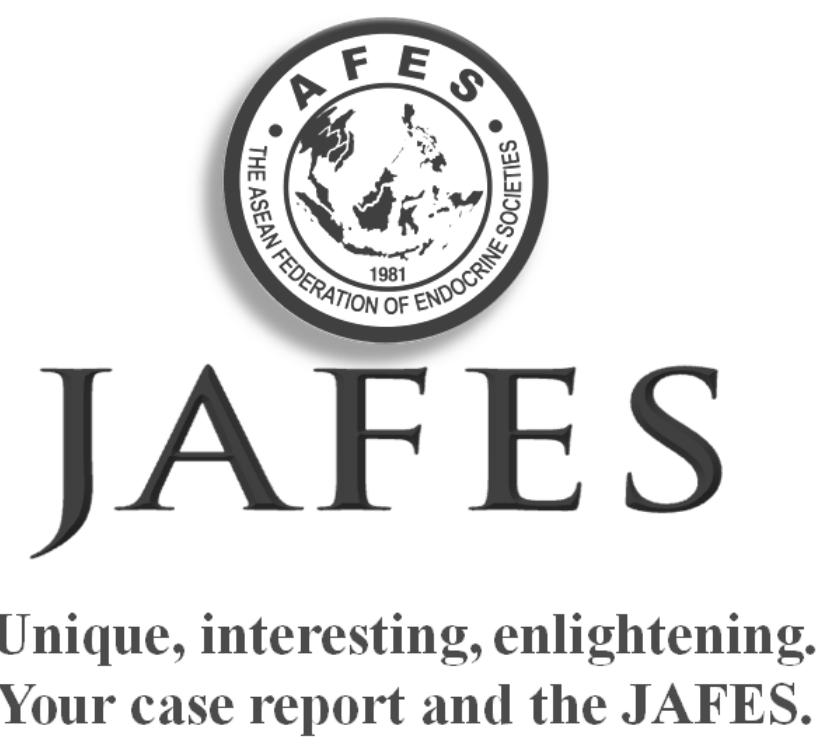

